Gastroenterologe 2010 · 5:207-208

DOI 10.1007/s11377-010-0439-3

(c) Springer-Verlag 2010

\author{
R. Jakobs ${ }^{1}$. J.F. Riemann ${ }^{2}$ \\ ${ }^{1}$ Medizinische Klinik C, Klinikum der Stadt Ludwigshafen \\ ${ }^{2}$ Ludwigshafen
}

\title{
Interdisziplinarität in der Gastroenterologie
}

Die medizinischen und wirtschaftlichen Entwicklungen der letzten Jahrzehnte fordern in allen Disziplinen ein vernetztes Denken, gute Kommunikation und enge fachliche Abstimmung zwischen Ärzten und anderen Leistungserbringern. Die Spezialisierung in den einzelnen Fachbereichen hat dazu beigetragen, dass selbst innerhalb eines Fachgebiets „Generalisten“, die noch die ganze Breite ihres Gebiets beherrschen, seltener geworden sind. Doch gerade in Problemsituationen, die potenziell fachliche Grenzzonen betreffen, ist eine intensive interdisziplinäre $\mathrm{Zu}$ sammenarbeit unabdingbar. Diese Ausgabe des „Gastroenterologen“ wurde deshalb besonders unter dem Aspekt der Interdisziplinariät in der Gastroenterologie zusammengestellt und beleuchtet spezielle Abstimmungsbereiche unseres Fachgebiets.

Der erste Beitrag ist ein Erfahrungsbericht über die erste interdisziplinäre gastroenterologisch-viszeralchirurgische Station in Deutschland, die in der Folgezeit als Modell an verschiedenen Kliniken frühzeitig auch in unserer Abteilung - in unterschiedlichen Varianten nachempfunden wurde. Eine gemeinsame „Bauchstation“ oder „Zentren für Viszeralmedizin“ sind mittlerweile in vielen Krankenhäusern entstanden, manchmal nur unter Marketingaspekten. Doch nur selten wird überprüft, ob sie tatsächlich so strukturiert sind, dass sie zu einer Verbesserung der Versorgungsqualität einerseits und zu besseren Erlösen andererseits beitragen. Der Artikel ist kein wissenschaftlicher Report, sondern steht exemplarisch für das Auf und $\mathrm{Ab}$ der interdisziplinären Modellstationen unter den zunehmenden
Zwängen des DRG-Systems und einer sich rasch wandelnden Kliniklandschaft.

Die klassische Domäne der CED-Diagnostik war in den letzten Jahren die Endoskopie. Mit der Ballonenteroskopie und der Kapselendoskopie konnten Lücken in der Dünndarmdarstellung geschlossen werden. Neue Methoden der Radiologie und der MRT, aber auch eine Reihe an Labortests, ermöglichen auch weniger invasiv wichtige Informationen zur Diagnose und Verlaufsbeurteilung. Wo sie die endoskopische Diagnostik ersetzen oder sogar bessere Ergebnisse erzielen, wird kritisch diskutiert.

\section{จ Vernetztes Denken, gute Kommunikation und enge fachliche Abstimmung sind gefordert}

Die Adipositas ist insbesondere in den westlichen Ländern eines der Hauptprobleme des 21. Jahrhunderts. Die resultierenden Folgeerkrankungen, von Diabetes über Fettleber (und Leberzirrhose) bis hin zu orthopädischen Problemen, werden zur massiven Belastung für die Gesundheitssysteme. Mit den Methoden bariatrischer Operationen scheint bei morbider Adipositas ein sehr effektiver Weg zur Therapie gefunden zu sein. Der viszeralchirurgische Beitrag spiegelt die langjährige Erfahrung der Autoren gerade für diese Operationsverfahren wieder. Für wen sie in Frage kommen, welche Probleme $\mathrm{zu}$ erwarten sind und ob es bereits weniger invasive Alternativen durch endoskopische Maßnahmen gibt, wird dankenswerterweise in einem Beitrag der Brüsseler Arbeitsgruppe um Jacques Deviére, einer international renommierten endoskopischen Spitzengruppe, dargestellt.

Mit der rasanten Entwicklung und Verbreitung interventioneller endoskopischer Eingriffe am Gastrointestinaltrakt, aber auch der Ausweitung großer onkologischer Operationen steigt die Rate an Komplikationen. In diesem Spannungsfeld der Viszeralmedizin ist zwischen Viszeralchirurgen und Gastroenterologen eine besonders intensive Diskussion und Zusammenarbeit erforderlich, um ein optimales Komplikationsmanagement zu gewährleisten. Die typischen Komplikationen und deren derzeitige endoskopische wie chirurgische Behandlungsoptionen werden unter aktuellen Gesichtspunkten erläutert.

Ein weiterer Beitrag befasst sich mit dem Grenzbereich GastroenterologieViszeralchirurgie in der lokalen Ablation von Frühkarzinomen im Gastrointestinaltrakt. Wie ist ein optimiertes Vorgehen zu erreichen, wo ist die endoskopische Abtragung noch möglich, wann ist ein kombiniertes endoskopisch-chirurgisches Vorgehen sinnvoll? Antworten und Empfehlungen finden Sie im Artikel.

Der Zusammenhang zwischen Thoraxschmerz und Herzinfarkt (oder zumindest koronarer Herzkrankheit) wird von vielen Medizinern reflexartig hergestellt. Dass diese Symptomatik ein sehr viel differenzierteres Vorgehen und differenzialdiagnostisches Denken erfordert, wird abschließend gezeigt. Es handelt sich um ein praxisorientiertes Review, das nicht nur für die Notaufnahme, sondern auch für die Kollegen in der niedergelassenen Praxis und auf Normalstation von Interesse ist. 
Wir sind sicher, dass es uns mit der Zusammenstellung der Beiträge gelungen ist, Ihnen wichtige Bereiche der heute zwangsläufigen und sinnvollen Interdisziplinarität zwischen Gastroenterologie und benachbarten Disziplinen darzustellen, vor allem der Viszeralchirurgie, aber auch - von manchen nicht unbedingt an dieser Stelle erwartet - der Kardiologie. Wir hoffen, dass Sie bei der Lektüre dieser Ausgabe des „Gastroenterologen“ viele Anregungen für ihre tägliche Arbeit und zur fachlichen Diskussion finden. Die Deutsche Gesellschaft für Verdauungsund Stoffwechselkrankheiten (DGVS) trägt dieser Entwicklung bekanntlich inzwischen dadurch Rechnung, dass sie ihren Jahreskongress zusammen mit der Deutschen Gesellschaft für Viszeralchirurgie (DGVC) als gemeinsame Jahrestagung für Viszeralmedizin ausrichtet. Auch im Interesse des Patienten ist das eine außerordentlich begrüßenswerte und sicher längst fällige Entwicklung.<smiles>CCCCCC(=O)CCCC</smiles>

R. Jakobs<smiles>CC(C)CC1CC1</smiles>

J.F. Riemann

\section{Korrespondenzadressen}

\section{Prof. Dr. R. Jakobs}

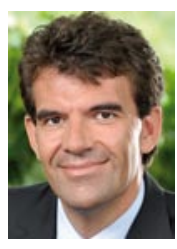

Medizinische Klinik C, Klinikum der Stadt Ludwigshafen Bremserstr. 79, 67063 Ludwigshafen jakobsr@klilu.de

\section{Prof. Dr. J.F. Riemann}

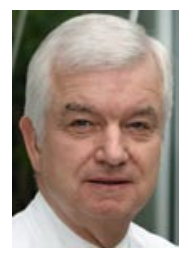

Der Gastroenterologe bietet umfassende und aktuelle Beiträge zu interessanten Themenschwerpunkten aus allen Bereichen der Gastroenterologie und Hepatologie.

Möchten Sie ein bereits erschienenes Heft nachbestellen? Die folgenden Ausgaben aus 2009 und bereits erschienene Ausgaben aus 2010 können Sie direkt bei unserem Kundenservice zum Preis von EUR 32,- beziehen:

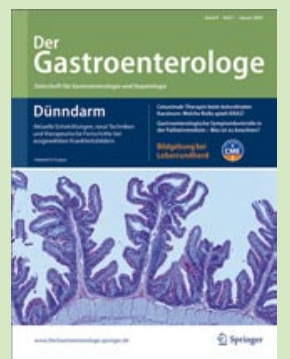

\section{9}

- 1/09 Dünndarm

- 2/09 Autoimmune Lebererkrankungen

- 3/09 Ösophagus- und Magenkarzinom

- 4/09 Viszeralmedizin - komplexe Krankheitsbilder aus hepatologischer und chirurgischer Sicht

- 5/09 Viszeralmedizin - komplexe Krankheitsbilder aus gastroenterologischer und chirurgischer Sicht

- 6/09 Leber und Pankreas

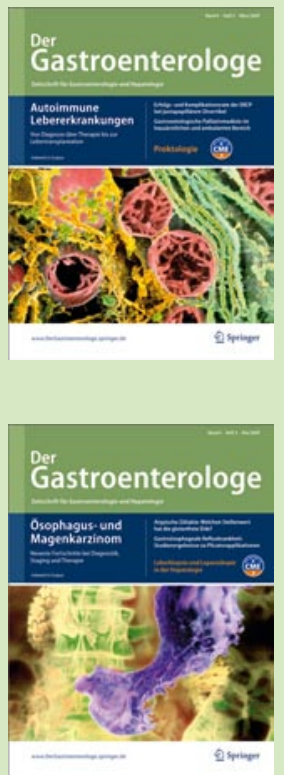

\section{0}

- 1/10 Hereditäre gastroenterologische Erkrankungen

- 2/10 NASH \& Co.

- 3/10 Interdisziplinäre Gastroenterologie

- 4/10 Proktologie

- 5/10 Gastrointestinale Onkologie

- 6/10 Bildgebung in der Gastroenterologie

\section{(Änderungen vorbehalten)}

So erreichen Sie unseren Kundenservice:

Springer Medizin Verlag GmbH

Kundenservice Zeitschriften

Haberstraße 7

69126 Heidelberg

Tel. $+496221345-4303$

Fax: +49 6221 345-4229

E-Mail: subscriptions@springer.com

www.DerGastroenterologe.springer.de 